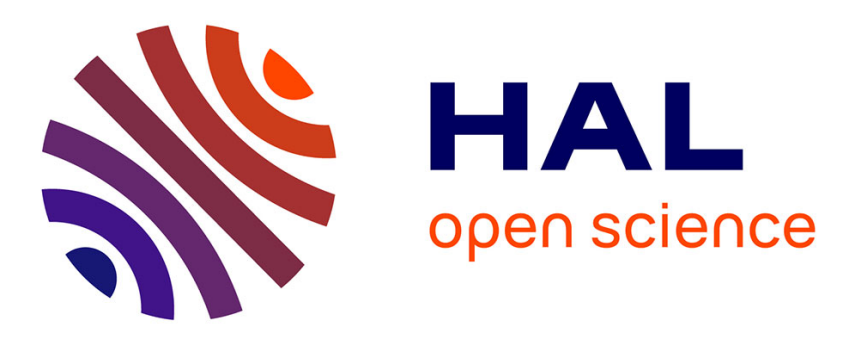

\title{
Attribute Controlled Reconstruction and Adaptive Mathematical Morphology
}

Andrés Serna, Beatriz Marcotegui

\section{To cite this version:}

Andrés Serna, Beatriz Marcotegui. Attribute Controlled Reconstruction and Adaptive Mathematical Morphology. 11th International Symposium, ISMM 2013, May 2013, Uppsala, Sweden. pp.207-218, 10.1007/978-3-642-38294-9_18. hal-00834638

\section{HAL Id: hal-00834638 \\ https://hal-mines-paristech.archives-ouvertes.fr/hal-00834638}

Submitted on 17 Jun 2013

HAL is a multi-disciplinary open access archive for the deposit and dissemination of scientific research documents, whether they are published or not. The documents may come from teaching and research institutions in France or abroad, or from public or private research centers.
L'archive ouverte pluridisciplinaire HAL, est destinée au dépôt et à la diffusion de documents scientifiques de niveau recherche, publiés ou non, émanant des établissements d'enseignement et de recherche français ou étrangers, des laboratoires publics ou privés. 


\title{
Attribute controlled reconstruction and adaptive mathematical morphology
}

\author{
Andrés Serna and Beatriz Marcotegui \\ \{andres.serna_morales, beatriz.marcotegui\}@mines-paristech.fr \\ Mines ParisTech \\ CMM - Centre de Morphologie Mathématique \\ Mathématiques et Systèmes \\ 35 rue St Honoré 77305, Fontainebleau-CEDEX, France
}

\begin{abstract}
In this paper we present a reconstruction method controlled by the evolution of attributes. The process begins from a marker, propagated over increasing quasi-flat zones. The evolution of several increasing and non-increasing attributes is studied in order to select the appropriate region. Additionally, the combination of criteria can be used in a straightforward way.

We present three applications on image segmentation, adaptive mathematical morphology and feature extraction. Firstly, our method successfully segments connected objects in range images. Secondly, inputadaptive structuring elements (SE) are defined computing the propagation approach for each pixel on a pilot image. Finally, input-adaptive SE are used to assess shape features on the image.

Compared with other methods, our approach is multi-scale and autodual, it does no require parameter to determine the appropriate region, and it is useful to extract objects of a given shape. Additionally, our reconstruction is a connected operator since quasi-flat zones does not create new contours on the image.
\end{abstract}

Keywords: mathematical morphology, controlled reconstruction, connected operators, adaptive SE, quasi-flat zones, attribute evolution.

\section{Introduction}

Local operators constitute powerful techniques in digital image processing. They are based on the neighborhood of each pixel, defined by a kernel. In general, a kernel is a ball of radius $r$ centered at the point to be processed. In the digital case, the kernel is reduced to the definition of a local neighborhood describing the connections between adjacent pixels. In Mathematical Morphology (MM), these kernels are called structuring elements (SE) and they are the base of sophisticated nonlinear techniques for filtering, feature extraction, detection and segmentation $[1,2,3]$.

In practice, square SE are preferred. However, several works remark the usefulness and necessity of adapting algorithms according to intrinsic variability and 
a priori knowledge of the image [4]. Adaptive structuring elements are elegant processing techniques using non-fixed kernels. Such operators, firstly introduced by Gordon and Rangayyan [5], vary their shape over the whole image taking into account local image features. Serra [3] called them structuring functions and defined erosion and dilation with spatially-varying SE.

In the literature, several works have been carried out with the aim of using image information in order to locally adapt SE shape and size. An overview on adaptive MM can be found in [4]. Most works proposed filters that privilege smoothing in homogeneous regions while preserving edges as well as possible. With this idea, Perona and Malik [6] proposed anisotropic filters that inhibit diffusion through strong gradients.

One of the first works using adaptive SE is due to Beucher [7]. He developed a traffic control application where the SE size depended on the perspective and varied linearly with the vertical position of the vehicle on a video sequence. Later, Verly and Delanoy [8] applied adaptive MM to range imagery. Since range images contain significant shape information, adaptive MM is a natural way to deal with shapes while correcting perspective distortions. Their approach consists in defining square SE such that their size depends on the distance between objects and sensor. Shih and Cheng [9] used simple and fast adaptive dilations with elliptic SE that varies its size and orientation according to local properties. A more sophisticated solution, proposed by Talbot and Appleton [10], defines pixel connectivities by complete and incomplete paths. Pinoli and Debayle [11] proposed a general adaptive neighborhood for MM: given a criterion mapping $h$ and a tolerance $m>0$, at each point $x$ an adaptive neighborhood is defined containing all points $y$ such that $|h(y)-h(x)|<m$. Lerallut et al. [12] proposed adaptive SE, called amoebas. These amoebas take image gradient into account in order to adapt their shape. Morard et al. [13] proposed adaptive SE based on a region growing process. These SE have a fixed size but they adapt their shape by choosing recursively homogeneous pixels with respect to the seed pixel. Angulo [14] used the notion of counter-harmonic mean in order to propose bilateral filters which assimtotically correspond to spatially-variant morphological operators. Note that all these works are applied to MM, however they are useful to any other local operators such as convolution or non-linear filters.

In this work, a reconstruction method controlled by the evolution of a given attribute is presented (e.g. gray-level statistics, area, geodesic distances, among others). The process begins from markers, propagated over increasing quasi-flat zones, avoiding the creation of new contours on the image. Then, the best propagation is selected according to the attribute changes. We show that our method does no required attribute threshold in order to determine the appropriate region, it is multi-scale and auto-dual. To demonstrate its usefulness, applications in image segmentation, adaptive SE and feature extraction are presented.

This paper is organized as follows. Section 2 presents a background on connectivity relations and quasi-flat zones. Section 3 defines propagation controlled by the evolution of attributes. Section 4 illustrates three applications. And Section 5 concludes the paper. 


\section{Background}

Connectivity relations are equivalence relations that naturally lead to partitions satisfying all conditions of image segmentation, that is: "a segmentation is a partition into disjoint connected subsets (called segments) such that there exists a logical predicate returning true on each segment but false on any union of adjacent segments" [15]. For example, the connectivity relation induced by the equality of gray-level divides the image into maximal connected components of constant gray-level, called flat-zones [16]. In most cases, partition in flat zones results in too many segments. A less restrictive connectivity relation can be defined adding a threshold $\lambda$. It allows to connect adjacent pixels if their graylevel difference does not exceed $\lambda$. This procedure, first introduced in image processing by Nagao et al. [17], is called quasi-flat (or $\lambda$-flat) zones labeling and it is defined as [18]:

Definition 1. Let $f$ be a digital gray-scale image $f: D \rightarrow V$, with $D \subset Z^{2}$ the image domain and $V=[0, \ldots, R]$ the set of gray levels. Two neighboring pixels $p, q$ belong to the same $\lambda$-flat zone of $f$, if their difference $\left|f_{p}-f_{q}\right|$ is smaller than or equal to a given $\lambda$ value.

The definition of $\lambda$-flat zones is very useful in image partition, simplification and segmentation. However, it suffers from the well-known chaining effect of the single linkage clustering [19]. That is, if two distinct image objects are separated by one or more transitions going in steps having a gray-level difference lower than $\lambda$, they will be merged in the same $\lambda$-flat zone.

Several works try to restrict quasi-flat zones growth in order to prevent merging between different regions. For example, Hambrusch et al. [20] proposed a technique to limit the chaining effect by introducing an additional threshold that limits gray-level variation over the whole connected component rather than just along connected paths. This relation is reflexive and symmetric, but not necessarily transitive, so it does not always lead to an image partition in the definition domain. In [21], Soille reviewed several approaches and proposed a constrained connectivity called $(\lambda, \omega, \beta)$-connectivity. In this approach, a succession of $\lambda$-flat zones is built with increasing slope parameter $\lambda$ (up to a maximum $\left.\lambda_{\max }\right)$, none of which may have gray-level difference greater than $\omega$ and connectivity index greater than $\beta$. This method has the advantage of providing a unique partition of the image domain, which is very difficult to achieve in any other way. This method was successfully applied to hierarchical image partition and simplification. Other solutions include viscous propagations by means of geodesic reconstruction, as proposed in [22,23].

The main disadvantage of these approaches is how to tune the parameters. With the aim of simplifying this selection, we propose a non-parametric region growing approach based on increasing quasi-flat zones. It consists in evaluating attribute changes during region growing in order to select the appropriate partition. In that sense, our algorithm takes advantage of a priori knowledge and intrinsic information of the image. Let us explain it in the following section. 


\section{Attribute controlled reconstruction}

The idea comes from the reconstruction of an object from a marker. Let us describe the problem with the toy example of Figure 1. Consider a marker $x$ on the upper left corner of Figure 1(a) and its propagation by increasing $\lambda$-flat zones using 4-connected neighborhood. The propagation begins with $\lambda=0$ and it ends when propagation reaches the whole image at $\lambda=5$.

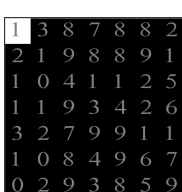

(a) $\lambda=0$

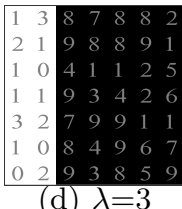

(d) $\lambda=3$

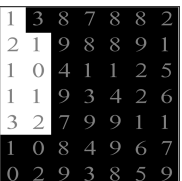

(b) $\lambda=1$

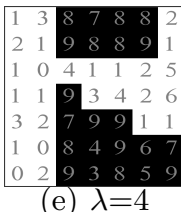

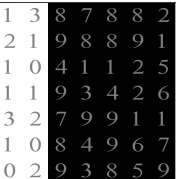

(c) $\lambda=2$

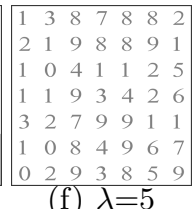

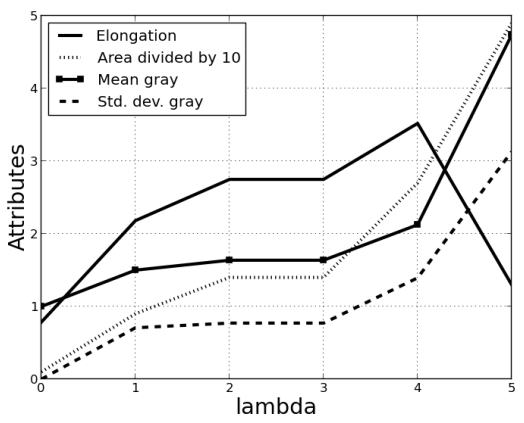

(g)

Fig. 1. Propagation over increasing $\lambda$-flat zones from a marker on the upper left corner.

In the domain of image segmentation, the question is: when should propagation be stopped? Obviously, the answer depends on the specific application. Intuitively, the evolution of an attribute could be useful to make the decision.

For example, Figure $1(\mathrm{~g})$ presents the evolution of four attributes: area $S(X)$, geodesic elongation ${ }^{1} E(X)$, mean gray-level $\mu_{f}(X)$ and standard deviation of gray-level $\sigma_{f}(X)$. We propose two criteria in order to select the propagation:

- Maximum attribute: To select the propagation such that the attribute is maximum.

- Attribute cataclysm: To select the propagation such that the attribute change between two consecutive $\lambda$ is maximum.

One one hand, one can see between $\lambda=3$ and $\lambda=4$ that area increases up to $200 \%$ of its value (from 14 to 27 pixels). This great change is called an attribute cataclysm, and it can be a reason to stop the growing process. Another example occurs between $\lambda=4$ and $\lambda=5$, where cataclysms are identified on $E(X), \mu_{f}(X)$ and $\sigma_{f}(X)$. On the other hand, the maximum elongation occurs in $\lambda=4$. Note that for increasing attributes (e.g. area) the maximum attribute value always corresponds to the propagation on the whole image. Therefore, selecting the

\footnotetext{
${ }^{1}$ For details on geodesic elongation definition, see Appendix A.
} 
maximum attribute is only reasonable in the case of non-increasing attributes (e.g. geodesic elongation). For further information about increasing and nonincreasing criteria, the reader is addressed to [24].

Based on Definition 1, let us introduce formal definitions for the set of increasing $\lambda$-flat zones:

Definition 2. For all $x \in D$, let $\Lambda_{x}$ be the set of increasing regions containing pixel $x$. For all $\lambda \in V$ and $j=[1, \ldots, n-1]$, we define $A_{x}(\lambda) \in \Lambda_{x}$ as the $\lambda$-flat zone of image $f$ containing $x$ :

$$
A_{x}(\lambda)=\{x\} \cup\left\{q \mid \exists \wp=\left(p_{1}=x, \ldots, p_{n}=q\right) \text { such that }\left|f_{p_{j}}-f_{p_{j+1}}\right| \leq \lambda\right\}
$$

In this work $\lambda$-flat zones are arbitrarily used. However, this is not a restrictive choice since any other hierarchical partition can be used as well. Let us introduce formal definitions for attribute cataclysm and maximum attribute:

Definition 3. Let $\Gamma\left(\Lambda_{x}\right)$ be an attribute on the family of increasing regions $\Lambda_{x}$ containing pixel $x$. We define the maximum attribute $M_{\Gamma}$ and the attribute cataclysm $C_{\Gamma}$ as:

$M_{\Gamma}=\operatorname{argmax}_{\lambda_{i} \in V}\left|\Gamma\left(A_{x}\left(\lambda_{i}\right)\right)\right|$

$C_{\Gamma}=\operatorname{argmax}_{\lambda_{i} \in V}\left|\Gamma\left(A_{x}\left(\lambda_{i}\right)\right)-\Gamma\left(A_{x}\left(\lambda_{i+1}\right)\right)\right|$

In this work, we arbitrarily analyze only one attribute at the same time. However, other statistics or combination of several attributes can be used as well. Compared with other methods, our main advantage is that no parameter is required in order to determine the adaptive region, it is a connected operator since the $\lambda$-flat zones do not create new contours on the image [16,25], it is multi-scale, and it is auto-dual since bright, dark and intermediate gray level regions are processed at the same time.

\section{Applications}

We present three applications related to image segmentation, input-adaptive SE and feature extraction: i) Reconstruction by controlled propagation from markers in order to segment connected objects in range images. ii) The controlled propagation is computed on a pilot image and the result is used as neighborhood of each pixel on the original image, similar to $[12,26]$. And, iii) Features can be computed on the input-adaptive SE and they are used to characterize each point on the image with respect to its neighborhood, similar to [13].

\subsection{Image segmentation}

Thanks to new 3D data availability, an increasing number of geographic applications such as Google Earth, Microsoft Virtual Earth, OpenStreetMaps and Geoportail is flourishing nowadays. Some of these applications do not only require to look realistic, but also have to be faithful to reality. Automatic urban structures segmentation is required in order to build accurate large scale 3D city models. In 
this section, we present an automatic facade segmentation method on 3D point clouds developed as part of TerraMobilita project (http://www.terramobilita.fr). It consists in four steps:

1. To project 3D data to a range image on a horizontal XY-plane (Figure 2(d)).

2. To find facade markers based on height constraints. Note that facades are the highest structures in the image.

3. To reconstruct facade from markers without including connected objects such as motorcycles parked next to them (Figure 2(c)).

4. To reproject the result to the 3D point cloud (Figure $2(\mathrm{~g})$ ).

For further details about steps 1, 2 and 4, the reader is referred to [27]. Let us concentrate on the third step since the other ones are straight-forward and are out of the scope of this paper. The attribute controlled reconstruction introduced in Section 3 is used for this purpose. Facades are elongated structures in the range image. Thus reconstruction stops when the geodesic elongation is maximum. Figure 2, where three motorcycles are parked next to the facade, exemplifies the process. Figures 2(a) and 2(b) show color pictures helpful to illustrate the scene. Figure 2(d) present the range image and the facade markers. Figure 2(f) shows the geodesic elongation evolution with increasing $\lambda$ values. Reconstruction at $\lambda=13$ is selected, which corresponds to the maximum elongation. Note that the maximum elongation (at $\lambda=13$ ) and the elongation cataclysm (at $\lambda=14$ ) are almost the same connected component, thus the stop criterion is not so critical for this example. The reconstruction result is shown in Figure 2(e) on the range image and in Figure 2(g) on the 3D point cloud. One can see that the entire facade is reconstructed correctly without including connected motorcycles.

With respect to other approaches in which a parameter should be selected, our method only requires selecting an attribute, then the appropriate propagation is automatically selected. This is useful when segmenting objects with similar attributes on large databases. For example, facades are always the most elongated structures. Then, if different $\lambda$ parameters are required to segment facades on different images (or even different facades on the same image), our method will adapts the parameter to the best possible value.

\subsection{Adaptive Morphology}

Among the different approaches in input-adaptive MM, amoebas [12] appear as a promising solution. They consist in defining a distance that depends on both the length and the gray-level differences on a neighborhood. This distance is used to define structuring elements $N(x)=\left\{y: d_{\sigma}(x, y) \leq r\right\}$ for each pixel on the input image. Because the amoeba distance is an increasing attribute, increasing $r$ leads to an inclusion property useful to define pyramid of operators [28]. In fact, if the process consists in successive operators (e.g. an opening is an erosion followed by a dilation), the SE should be the same for all of them in order to preserve mathematical properties of morphological filters, as proved by Roerdink [29]. Thus, adaptive SE are computed on a pilot image, the same for the whole process. 


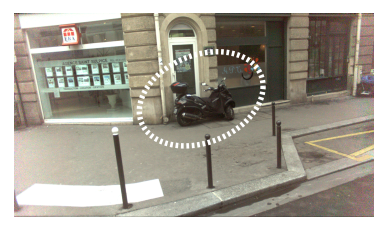

(a) Illustrative photo

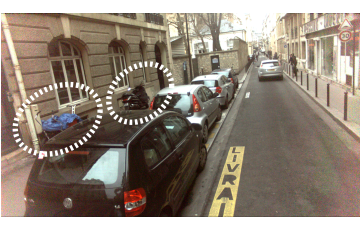

(b) Illustrative photo

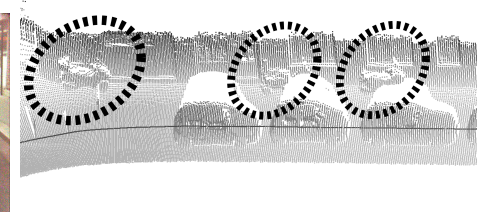

(c) 3D point cloud

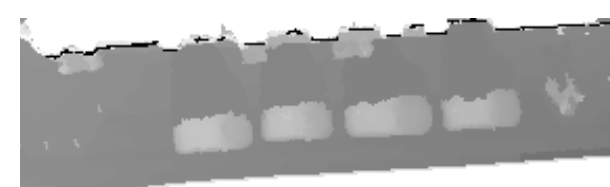

(d) Range image: facade markers (black)

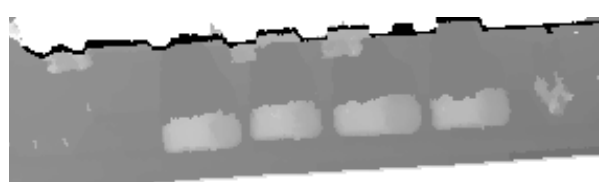

(e) Range image: reconstruction (black)

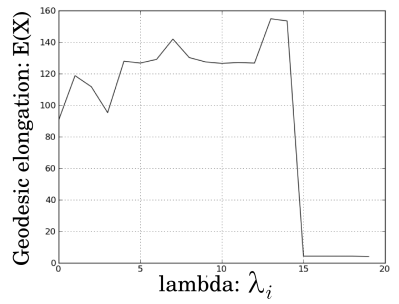

(f) Attribute evolution

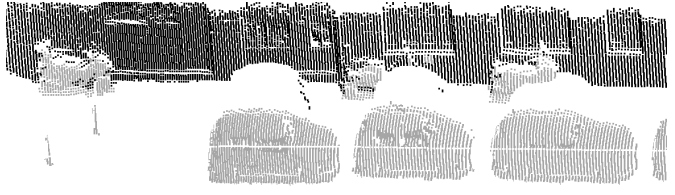

(g) Segmentated 3D point cloud

Fig. 2. Segmentation of connected objects by controlled propagation from markers.

This pilot image can be the original image or a filtered version of it since the noise can modify the SE shape.

In a similar way to amoebas, we apply our controlled propagation to define adaptive SE for each pixel on the pilot image. These adaptive SE are useful to filter structures according to a given attribute. With respect to amoebas, our method does no require parameters in order to define the SE shape.

For example, Figure 3 presents an opening with adaptive SE using the maximum elongation. Figure 3(c) illustrates the SE shape for two pixels in elongated and non-elongated regions. Figure 3(d) compares the result of an adaptive opening with respect to the classical one (Figure 3(b)). Note that elongated structures are preserved while non-elongated structures are merged with their neighborhood.

Figure 4(b) presents another example using gray-level cataclysm. This is useful to define SE containing pixels with similar gray-level. Figure 4(b) shows the SE shape for two different pixels in the image. Figure 4(c) presents the application of this adaptive SE as kernel of a non-linear filter, the median filter. Note that homogeneous regions are smoothed and high contrasted structures 


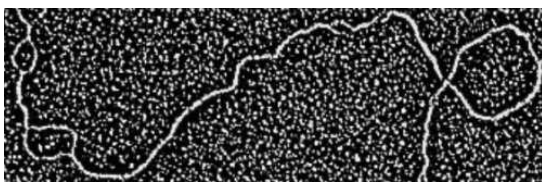

(a) Input image

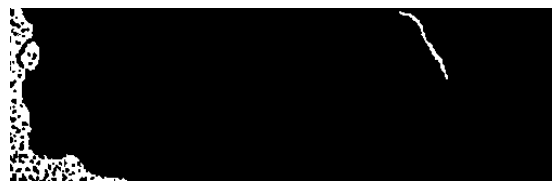

(c) Adaptive SE

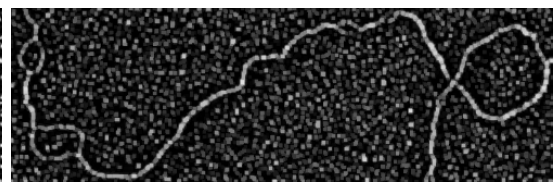

(b) Classic Opening

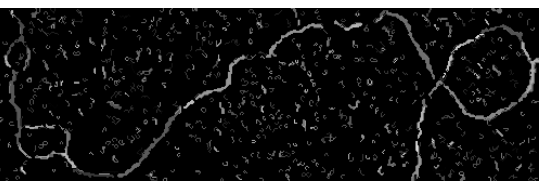

(d) Adaptive Opening

Fig. 3. Input-adaptive SE using the maximum elongation. In this case, the input and the pilot image are the same.

are preserved. Compared with amoebas and other similar works, our method is non-parametric since the SE size only depends on attribute selection.

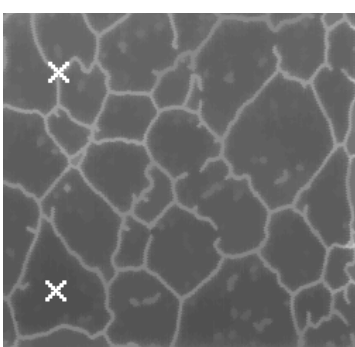

(a) Input image

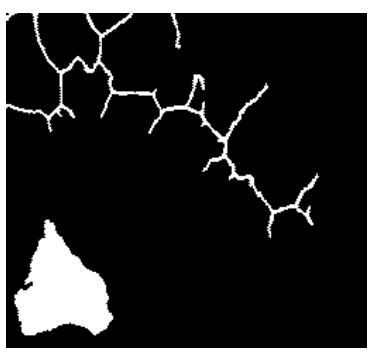

(b) Adaptive SE

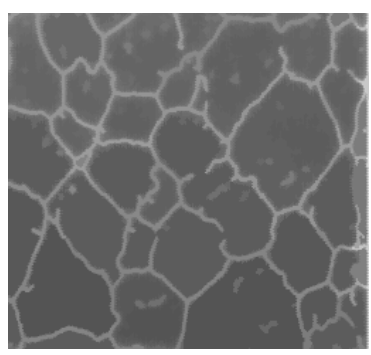

(c) Median Filter

Fig. 4. Input-adaptive SE using the gray-level cataclysm. White cross indicates the seed pixel. In this case, the input and the pilot image are the same.

\subsection{Feature Extraction}

We present an application to extract features from an image based on the shape of our input-adaptive SE. To the authors knowledge, this idea was first presented by Morard et al. [13], who proposed an approach using region growing structuring elements (REGSE). For each pixel on the image, they defined a neighborhood of $N$ pixels minimizing a homogeneity function $\rho(x)$ (e.g. gray-level difference) between adjacent pixels. REGSE can follow any homogeneous structure of a given size but cannot be multi-scale because all REGSE must have exactly $N$ 
pixels. Finally, they used the REGSE shape to compute shape features in the image.

We propose a similar approach with our propagation method. The main comparative advantage is that parameter $N$ is not required, because it is adaptively defined for each pixel during the propagation from it. In that sense, we use non-constant size SE that depends on the image intrinsic information. This is specially useful when the image contains objects at different scales. Additionally, remember that our propagation is a connected operator since $\lambda$-flat zones do not create new contours during propagation. This is not true for REGSE, where region growing is forced to stop at $N$ pixels.

Consider the four examples of Figure 5. From each pixel, we compute the adaptive SE using a propagation controlled by the maximal geodesic elongation. Each pixel on the output image contains the maximal geodesic elongation of its respective adaptive SE. Note that brighter and darker structures are processed at the same time. In order to favor one of them, feature image could be weighted using gray-level input image.

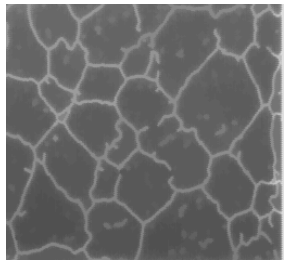

(a) Original image

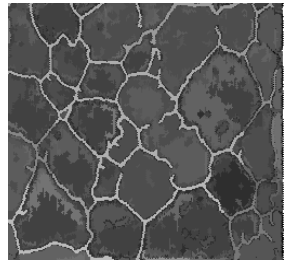

(b) Feature image

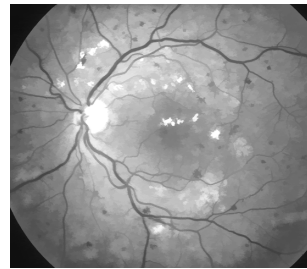

(c) Original image

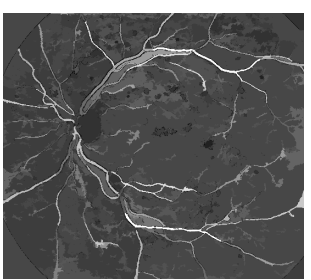

(d) Feature image

Fig. 5. Feature images using input-adaptive SE controlled by the maximal elongation.

Feature images are useful to assess features and segment structures by simple thresholding. Compared to geodesic thinnings [30] that uses geodesic elongation as our method does, our approach has the following advantages: i) Our feature image contains information about all objects in the scene, while geodesic thinning must be computed every time in order to extract structures at different elongations. ii) Our method, based on quasi-flat zones, deals with bright, dark or intermediate gray level regions at the same time whereas geodesic thinning, based on threshold decomposition of the image focuses only on bright objects. Figure 6 illustrates this comparison. Figures 6(b) and 6(c) present a geodesic thinning at $E(x)=11$ and $E(x)=20$, respectively. While Figures $6(\mathrm{e})$ and $6(\mathrm{f})$ present a simple thresholding on the feature image at these same values. Colored regions indicate different objects with elongation greater than selected threshold. 


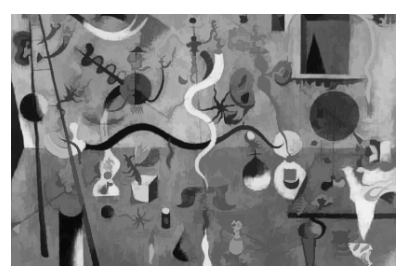

(a) Original image

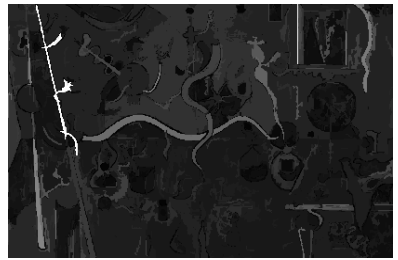

(d) Elongation image

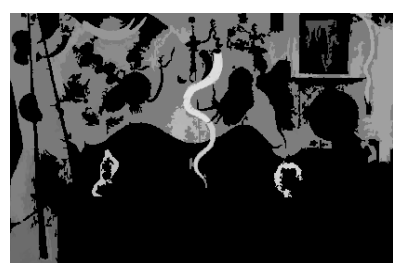

(b) Thinning at $E(x)=11$

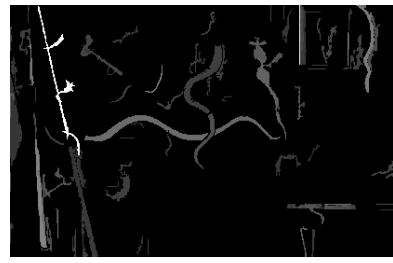

(e) Threshold at $E(x)=11$

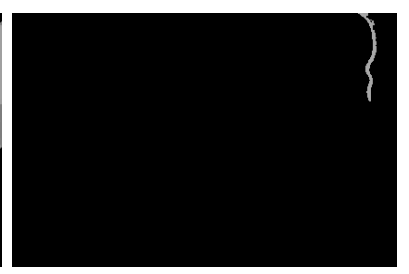

(c) Thinning at $E(x)=20$

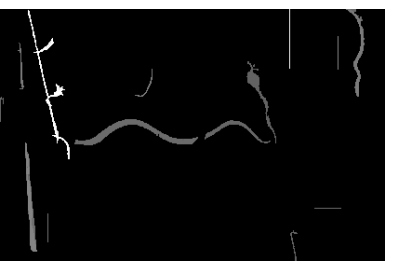

(f) Threshold at $E(x)=20$

Fig. 6. Extraction of elongated structures at different thresholds using geodesic thinnings [30] and thresholding on the feature image computed by our method.

\section{Conclusions}

We present a reconstruction method controlled by the evolution of a given attribute during propagation from markers. This method is a connected operator since the propagation is done on increasing quasi-flat zones, therefore new contours are not created. Any other connected hierarchical partition could be used while preserving this property. Additionally, our method is auto-dual since bright, dark and intermediate gray level regions are processed at the same time. When this controlled propagation is computed from each pixel on a pilot image, input-adaptive SE can be defined. The main advantage of our approach is that no attribute threshold is required in order to determine the appropriate region.

We present three applications on image segmentation, adaptive MM and feature extraction. In the first case, controlled propagation from markers is used to separate connected objects. In the second case, the propagation is computed from each pixel on a pilot image, then it is used to define input-adaptive SE that satisfies the properties of morphological filters. Moreover, if an increasing attribute is used, it satisfies the inclusion property necessary to define pyramid of operators. Finally, we take advantage of SE shape in order to assess features.

In this paper we have just studied the evolution of some attributes, but the idea can be extended to other attributes and even a combination of them in a straightforward way. In our experiments, attribute selection was done based on a priori knowledge.

Our propagation method is presented for gray-scales images. Future work will include its extension to color or multi-spectral images, where other metrics should be used to define propagation rules. 


\section{A Appendix: Geodesic elongation}

The geodesic elongation $E(X)$ of an object $X$, introduced by Lantuéjoul and Maisonneuve [31], is a shape descriptor useful to characterize long and thin structures. It is defined in Equation 1, where $S(X)$ is the area and $L(X)=$ $\sup _{x \in X}\left\{l_{x}(X)\right\}$ is the geodesic diameter [32], that means the longest geodesic arc of $\mathrm{X}$. The longer and narrower the object, the higher the elongation. The lower bound is reached with the disk, where $E(X)=1$. An efficient implementation can be found in [30].

$$
E(X)=\frac{\pi L^{2}(X)}{4 S(X)}
$$

\section{Acknowledgements}

This work has been performed as part of TerraMobilita project.

\section{References}

1. Matheron, G.: Random Sets and Integral Geometry. John Wiley \& Sons, New York (1975)

2. Serra, J.: Image Analysis and Mathematical Morphology. Volume 2. Academic Press, London (1988)

3. Serra, J.: Image Analysis and Mathematical Morphology: Theoretical Advance. Volume 2. Academic Press (1988)

4. Maragos, P., Vachier, C.: Overview of adaptive morphology: Trends and perspectives. (2009) 2241-2244

5. Gordon, R., Rangayyan, R.M.: Feature enhancement of film mammograms using fixed and adaptive neighborhoods. Applied Optics 23(4) (1984) 560-564

6. Perona, P., Malik, J.: Scale-space and edge detection using anisotropic diffusion. IEEE Transactions on Pattern Analysis and Machine Intelligence 12(7) (1990) 629-639

7. Beucher, S.: Traffic Spatial Measurements Using Video Image Processing. Intelligent Robots and Computer Vision 848 (1987) 648-655

8. Verly, J., Delanoy, R.: Adaptive mathematical morphology for range imagery. IEEE Transactions on Image Processing 2(2) (1993) 272-275

9. Shih, F.Y., Cheng, S.: Adaptive mathematical morphology for edge linking. Information Sciences 167(1) (2004) 9-21

10. Talbot, H., Appleton, B.: Efficient complete and incomplete path openings and closings. Image and Vision Computing 25(4) (2007) 416-425

11. Pinoli, J.c., Debayle, J.: General Adaptive neighborhood mathematical morphology. In: 16th IEEE International Conference on Image Processing (ICIP'09). (2009) 2249-2252

12. Lerallut, R., Decencière, É., Meyer, F.: Image filtering using morphological amoebas. Image and Vision Computing 25(4) (2007) 395-404

13. Morard, V., Decencière, E., Dokládal, P.: Region Growing Structuring Elements and New Operators based on their Shape. In: International conference on Signal and Image Processing (SIP'11), ACTA Press (2011) 
14. Angulo, J.: Morphological Bilateral Filtering and Spatially-Variant Adaptive Structuring Functions. In: Proceedings of the 10th International Symposium on Mathematical Morphology (ISMM'11). (2011) 212-223

15. Horowitz, S.L., Pavlidis, T.: Picture Segmentation by a directed split-and-merge procedure. Proceedings of the 2nd International Joint Conference on Pattern Recognition, Copenhagen, Denmark (1974) 424-433

16. Salembier, P., Serra, J.: Flat zones filtering, connected operators and filters by reconstruction. IEEE Transactions on Image Processing 4(8) (1995) 1153-1160

17. Nagao, M., Matsuyama, T., Ikeda, Y.: Region extraction and shape analysis in aerial photographs. Computer Graphics and Image Processing 10(3) (1979) 195223

18. Meyer, F.: From connected operators to levelings. In Heijmans, H., Roerdink, J., eds.: Mathematical Morphology and its Applications to Image and Signal Processing. Volume 12 of Computational Imaging and Vision., Kluwer Academic Publishers (1998) 191-198

19. Duda, R.O., Hart, P.E., Stork, D.G.: Pattern Classification. 2 edn. Wiley Interscience (2000)

20. Hambrusch, S., He, X., Miller, R.: Parallel Algorithms for Gray-Scale Digitized Picture Component Labeling on a Mesh-Connected Computer. Journal of Parallel and Distributed Computing 20(1) (1994) 56-68

21. Soille, P.: Constrained connectivity for hierarchical image decomposition and simplification. IEEE transactions on pattern analysis and machine intelligence 30(7) (2008) 1132-1145

22. Meyer, F., Vachier, C.: Image segmentation based on viscous flooding simulation. In Talbot, H., Beare, R., eds.: Proceedings of the 5th International Symposium on Mathematical Morphology (ISMM'02), Sydney, Australy (2002) 69-77

23. Serra, J.: Viscous Lattices. Journal of Mathematical Imaging and Vision 22 (2005) 269-282

24. Breen, E.J., Jones, R.: Attribute Openings, Thinnings, and Granulometries. Computer Vision and Image Understanding 64(3) (1996) 377-389

25. Salembier, P., Wilkinson, M.H.F.: Connected operators. IEEE Signal Processing Magazine 26(6) (2009) 136-157

26. Grazzini, J., Soille, P.: Adaptive Morphological Filtering Using Similarities Based on Geodesic Time. In Coeurjolly, D., Sivignon, I., Tougne, L., Dupont, F., eds.: Discrete Geometry for Computer Imagery. Volume 4992. Springer (2008) 519-528

27. Hernández, J., Marcotegui, B.: Point Cloud Segmentation towards Urban Ground Modeling. In: Joint Urban Remote Sensing Event, Urban 2009: 5th GRSS/ISPRS, Shangai, China (2009)

28. Serra, J., Salembier, P.: Connected operators and pyramids. SPIE Image Algebra and Morphological Image Processing 2030 (1993) 65-76

29. Roerdink, J.B.T.M.: Adaptivity and group invariance in mathematical morphology. In: Proceedings of the International Conference on Image Processing (ICIP'09), Cairo, Egypt (2009) 2253-2256

30. Morard, V., Decencière, E., Dokladal, P.: Geodesic attributes thinnings and thickenings. In: Proceedings of the 10th International Symposium on Mathematical Morphology (ISMM'11), Springer-Verlag (2011) 200-211

31. Lantuéjoul, C., Maisonneuve, F.: Geodesic methods in quantitative image analysis. Pattern Recognition 17(2) (1984) 177-187

32. Lantuéjoul, C., Beucher, S.: On the use of the geodesic metric in image analysis. Journal of Microscopy 121(1) (1981) 39-49 